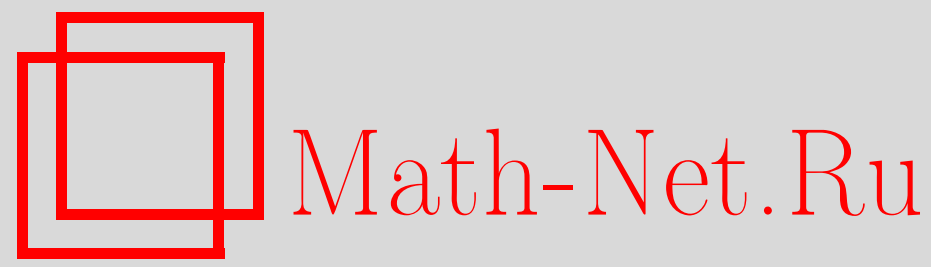

М. В. Семёнова, Вложение решеток в производные решетки, Совр. пробл. матем., 2011, выпуск 15, 67-82

DOI: https://doi.org/10.4213/spm29

Использование Общероссийского математического портала Math-Net.Ru подразумевает, что вы прочитали и согласны с пользовательским соглашением http://www . mathnet.ru/rus/agreement

Параметры загрузки:

IP : 52.205 .19 .152

26 апреля 2023 г., 17:52:07

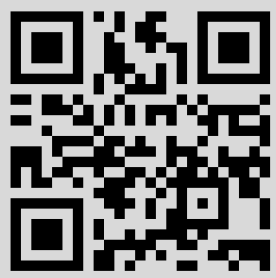




\title{
Вложение решеток в производные решетки
}

\author{
М. В. Семёнова \\ Институт математики им. С. Л. Соболева СО РАН, г. Новосибирск
}

Настоящая работа является расширенной версией доклада автора на Международной конференции "Мальцевские чтения", посвященной 100-летию со дня рождния академика А. И. Мальцева, которая прошла в г. Новосибирске 24-28 августа 2009 г. Речь здесь пойдет о недавних результатах, связанных с описанием классов решеток, вложимых в различные производные решетки, а также о некоторых нерешенных проблемах в этой области. В рамках статьи было бы довольно сложно дать обзор всего спектра проблем и результатов, касающихся рассматриваемых вопросов. Поэтому ограничимся упоминанием недавних результатов, полученных автором лично либо в соавторстве, а также результатов других авторов, тесно примыкающих к нашим.

\section{1. Введение}

Проблема вложения алгебраических систем в другие алгебраические системы, обладающие теми или иными свойствами, является классической в алгебре и теории моделей. Исследования в этой области были начаты А.И. Мальцевым в 30-е годы прошлого столетия в его пионерских работах [1], [2]. В этих работах Анатолий Иванович, в частности, показал, что класс полугрупп, вложимых в группы, является квазимногообразием (другими словами, может быть задан некоторым множеством квазитождеств в классе всех полугрупп). Кроме этого, он указал конкретный бесконечный базис квазитождеств для этого квазимногообразия, а также показал, что оно не может быть определено никаким конечным множеством квазитождеств (т.е. не является конечно базируемым).

В своей более поздней работе 1966 г. [3] Анатолий Иванович показал, что этот результат о вложимости полугрупп в группы является частью более общей картины. А именно, из характеризационной теоремы для квазимногообразий, доказанной в [3] (см. также [4]), вытекает, что если некоторый класс алгебраических систем (далее - просто систем) $\mathscr{K}$ замкнут относительно фильтрованных произведений, то класс систем, вложимых в системы из $\mathscr{K}$, является квазимногообразием. Для более точной формулировки этих результатов дадим необходимые определения.

Всюду далее, когда мы говорим о классе алгебраических систем, мы подразумеваем абстрактный (т.е. замкнутый относительно изоморфных копий) класс систем произвольной фиксированной сигнатуры. Класс алгебраических систем называется многообразием, если он определяется в классе всех систем того же типа тождествами, т.е. предложениями вида

$$
\forall \bar{x} \quad A_{0}(\bar{x}) \& \cdots \& A_{n}(\bar{x}),
$$

где $A_{0}(\bar{x}), \ldots, A_{n}(\bar{x})$ - атомарные формулы.

Класс систем называется квазимногообразием, если он определяется в классе всех систем того же типа квазитождествами, т.е. предложениями вида

$$
\forall \bar{x} \quad A_{0}(\bar{x}) \& \cdots \& A_{n}(\bar{x}) \rightarrow A(\bar{x}),
$$

где $A_{0}(\bar{x}), \ldots, A_{n}(\bar{x}), A(\bar{x})$ - атомарные формулы.

Работа выполнена при поддержке программы "Ведущие научные школы" (грант № НШ-3669.2010.1) и программы поддержки молодых ученых (грант № МД-2587.2010.1). 
Далее, для произвольного класса $\mathscr{K}$ пусть $\mathbf{V}(\mathscr{K})$ обозначает наименьшее многообразие, содержащее $\mathscr{K}$ (другими словами, многообразие, порожденное $\mathscr{K})$, а $\mathbf{Q}(\mathscr{K})$ - квазимногообразие, порожденное $\mathscr{K}$. Кроме того, пусть $\mathbf{P}(\mathscr{K})$ обозначает класс систем, изоморфных декартовым произведениям систем из $\mathscr{K} ; \mathbf{P}_{r}(\mathscr{K})-$ класс систем, изоморфных фильтрованным произведениям систем из $\mathscr{K} ; \mathbf{P}_{u}(\mathscr{K})$ - класс систем, изоморфных ультрапроизведениям систем из $\mathscr{K} ; \mathbf{S}(\mathscr{K})$ - класс систем, изоморфных подсистемам систем из $\mathscr{K}$ (другими словами, класс систем, изоморфно вложимых или просто вложимых в системы из $\mathscr{K}) ; \mathbf{H}(\mathscr{K})-$ класс гомоморфных образов систем из $\mathscr{K}$, а $\mathscr{K}_{\text {fin }}$ обозначает класс конечных систем из $\mathscr{K}$. В терминах операторов над классами упомянутая выше характеризационная теорема Мальцева выглядит следующим образом.

Теорема 1.1 (Мальцев [3]). Для произвольного класса систем $\mathscr{K}$ имеют место равенства $\mathbf{Q}(\mathscr{K})=\mathbf{S P}_{r}(\mathscr{K})=\mathbf{S P P}_{u}(\mathscr{K})$.

Для многообразий характеризационная теорема была доказана Биркгофом в 1935 г.

Теорема 1.2 (Биркгоф [5]). Для произвольного класса систем $\mathscr{K}$ имеют место равенства $\mathbf{V}(\mathscr{K})=\mathbf{H S P}(\mathscr{K})$.

Удобные термины для формулировки различных проблем вложения в классе решеток дают понятия пространства замыкания и решетки замыканий.

Пространством замыкания называется пара $(X, \varphi)$, где $X$ - множество с определенным на нем оператором замыкания $\varphi$, который удовлетворяет следующим условиям:

(1) $A \subseteq \varphi(A) \subseteq X$ для любого $A \subseteq X$;

(2) $\varphi^{2}(A)=\varphi(A)$ для любого $A \subseteq X$;

(3) $\varphi(A) \subseteq \varphi(B)$ для любых $A \subseteq B \subseteq X$.

Если $\varphi(A)=A$, то множество $A \subseteq X$ называется замкнутым. Множество

$$
\mathrm{Cl}(X, \varphi)=\{A \subseteq X \mid A=\varphi(A)\}
$$

всех замкнутых подмножеств в $(X, \varphi)$ образует полную решетку относительно включения, в которой для любого семейства $A_{i} \in \mathrm{Cl}(X, \varphi), i \in I$,

$$
\bigwedge_{i \in I} A_{i}=\bigcap_{i \in I} A_{i}, \quad \bigvee_{i \in I} A_{i}=\varphi\left(\bigcup_{i \in I} A_{i}\right) \text {. }
$$

Решетку $\mathrm{Cl}(X, \varphi)$ мы называем также решеткой замыканий.

Пусть $A$ - алгебраическая система. Для любого $B \subseteq A$ пусть $\operatorname{Sub}(B)$ обозначает подсистему в $A$, порожденную множеством $B$. Тогда $(A, \mathrm{Sub})$ является пространством замыкания, для которого решетка замыканий совпадает с решеткой подсистем $\operatorname{Sub}(A)$. Другим примером решеток замыканий являются решетки квазимногообразий. Пусть $\mathscr{Q}$ - квазимногообразие. Напомним, что для любого подкласса $\mathscr{A}$ в $\mathscr{Q}$ наименьшее квазимногообразие, содержащее $\mathscr{A}$, мы обозначаем через $\mathbf{Q}(\mathscr{A})$. Тогда решетка подквазимногообразий в $\mathscr{Q}$ является решеткой замыканий для $(\mathscr{Q}, \mathbf{Q})$.

Конечно, два приведенных примера не исчерпывают весь список решеток замыканий, которые служат объектом широкого круга исследований во многих областях универсальной алгебры, теории моделей, теории решеток (см. [6], [7]), связанных с изучением свойств решеток замкнутых подмножеств как конкретных пространств замыканий, так и абстрактных пространств замыканий в целом. В этих исследованиях естественным образом возникают следующие проблемы.

- Для заданного класса $\mathscr{C}$ пространств замыкания описать класс $\mathbf{S ~ C l}(\mathscr{C})$ решеток, вложимых в решетки замыканий пространств из $\mathscr{C}$.

- Является ли класс $\mathbf{S ~ C l}(\mathscr{C})$ аксиоматизируемым на языке первого порядка? 
- Является ли класс $\mathbf{S ~ C l}(\mathscr{C})$ (квази)многообразием?

- Описать класс $\mathbf{S ~ C l}(\mathscr{C})_{\text {fin }}$ конечных решеток, вложимых в решетки замыкания пространств из $\mathscr{C}$.

$\mathrm{K}$ настоящему времени наиболее изучены два типа пространств замыкания: комбинаторные геометрии (Корте, Ловас, Шрадер [8]) и выпуклые геометрии (Адаричева, Горбунов, Туманов [9]).

\section{2. Выпуклые геометрии}

Пространство замыкания $(X, \varphi)$ называется выпуклой геометрией, если оно обладает следующим свойством антизамены:

$$
a \in \varphi(Y \cup\{b\}), \quad a \notin \varphi(Y) \quad \text { влечет } \quad b \notin \varphi(Y \cup\{a\})
$$

для любых $a \neq b$ из $X$ и любого $Y \subseteq X$. Свойство антизамены является обобщением естественного понятия выпуклости в афинных пространствах (см. рис. 1).

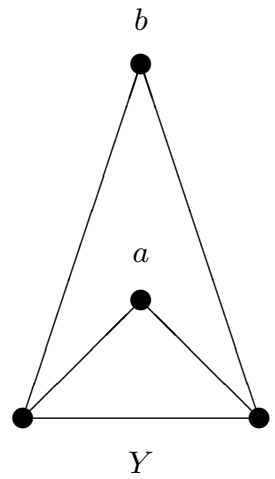

Рис. 1. Иллюстрация свойства антизамены

Решетка замыкания любой конечной выпуклой геометрии полудистрибутивна вверх, т.е. удовлетворяет квазитождеству

$$
\forall x, y, z \quad x \vee y=x \vee z \rightarrow x \vee y=x \vee(y \wedge z)
$$

Пусть $\mathrm{SD}^{\vee}$ обозначает класс полудистрибутивных вверх решеток. Очевидно, свойство полудистрибутивности вверх устойчиво при переходе к подрешеткам, поэтому любая подрешетка решетки замыканий конечной выпуклой геометрии полудистрибутивна вверх. Оказывается, что класс конечных выпуклых геометрий универсален для класса конечных полудистрибутивных вверх решеток в следующем смысле.

Теорема 2.1 (Адаричева, Горбунов, Туманов [9]). Конечная решетка полудистрибутивна вверх тогда и только тогда, когда она вложима в решетку замыканий некоторой конечной выпуклой геометрии. Другими словами, $\mathrm{SD}_{\mathrm{fin}}^{\vee}=\mathbf{S ~ C l}(\mathscr{C})$, где $\mathscr{C}$ обозначает класс конечных выпуклых геометрий.

В связи с этим результатом была поставлена

Проблема 1 (Адаричева, Горбунов, Туманов [9]). Существует ли класс $\mathscr{U}$ конечных выпуклых геометрий конкретного вида, универсальный для класса всех конечных полудистрибутивных вверх решеток (другими словами, класс $\mathscr{U}$, для которого справедливо равенство $\left.\operatorname{SCl}(\mathscr{U})=\mathrm{SD}_{\text {fin }}^{\vee}\right) ?$ 
Далее мы рассмотрим проблему 1 в контексте некоторых конкретных пространств замыкания.

2.1. Выпуклые геометрии в векторных пространствах. Для произвольного тела $\mathbb{D}$ и натурального $n>0$ пусть $\mathbb{D}_{\mathbb{D}}^{n}$ обозначает $n$-мерное векторное пространство над $\mathbb{D}$. Если $\mathbb{D}$ - линейно упорядоченное тело, а $\left\langle V_{\mathbb{D}},+,-, \mathbb{D}\right\rangle$ - векторное пространство над $\mathbb{D}$, то множество $U \subseteq V_{\mathbb{D}}$ называется выпуклым, если $\lambda x+(1-\lambda) y \in U$ для любых $x, y \in U$ и любого $\lambda \in[0,1] \subseteq \mathbb{D}$. Для $X \subseteq V_{\mathbb{D}}$ пусть $\mathrm{Co}(X)$ обозначает наименышее выпуклое подмножество в $V_{\mathbb{D}}$, содержащее $X$ (или выпуклую оболочку множества $X$ ). Соответствующую решетку замкнутых (выпуклых в данном контексте) подмножеств мы обозначаем через $\mathrm{Co}\left(V_{\mathbb{D}}\right)$.

Если $X \subseteq V_{\mathbb{D}}$, то полагаем

$$
\operatorname{Co}_{X}\left(V_{\mathbb{D}}\right)=\left\{X \cap Y \mid Y \in \operatorname{Co}\left(V_{\mathbb{D}}\right)\right\}
$$

и любое множество $U \in \mathrm{Co}_{X}\left(V_{\mathbb{D}}\right)$ называем относительно выпуклым в $V_{\mathbb{D}}$. Нетрудно видеть, что для любого $X \subseteq V_{\mathbb{D}}$ пространство замыкания $\left(X, \mathrm{Co}_{X}\right)$ является выпуклой геометрией, a $\mathrm{Co}_{X}\left(V_{\mathbb{D}}\right)$ - ее решеткой замкнутых (выпуклых) подмножеств.

В связи с проблемой 1 ее авторы поставили также следующий вопрос.

Проблема 2 (Адаричева, Горбунов, Туманов [9]). Является ли класс конечных решеток относительно выпуклых подмножеств векторных пространств универсальным для класса $\mathrm{SD}_{\text {fin }}^{\vee}$ ?

Частично (положительный) ответ на этот вопрос дают теоремы 2.2 и 2.3 , но по сути проблема 2 до сих пор остается открытой.

Теорема 2.2 (Верунг, Семенова [10]). Пусть $\mathbb{D}$ - линейно упорядоченное тело. Для любой решетки $L$ существует вектороное пространство $V_{\mathbb{D}}$ над $\mathbb{D}$, такое что $L$ вложима в $\mathrm{Co}\left(V_{\mathbb{D}}\right)$.

Для формулировки следующей теоремы дадим необходимые определения. Решеточный гомоморфизм $h: K \rightarrow L$ ограничен снизу, если для любого $a \in L$ множество $\{x \in K \mid h(x) \geqslant a\}$ либо пусто, либо содержит наименьший элемент. Решетка $L$ называется ограниченной снизу, если для любой конечно порожденной решетки $K$ любой гомоморфизм $h: K \rightarrow L$ ограничен снизу. Понятие ограниченного (снизу) гомоморфизма служит обобщением понятия $(\wedge)$-полного гомоморфизма и впервые появилось в работе Маккензи [11] в связи с изучением немодулярных многообразий решеток. Это понятие нашло естественные приложения и в теории свободных решеток, где был получен ряд замечательных результатов (см. монографию Фриза, Ежека, Нейшна [12]).

Пусть $L B$ обозначает класс ограниченных снизу решеток. Отметим, что класс $L B_{\text {fin }}$ конечных ограниченных снизу решеток образует собственный подкласс в классе конечных полудистрибутивных вверх решеток и порождает многообразие всех решеток. Для класса $L B_{\text {fin }}$ ответ на вопрос, поставленный в проблеме 2, положителен.

Теорема 2.3 (Верунг, Семенова [10]). Пусть $\mathbb{D}$ - линейно упорядоченное тело. Любая конечная ограниченная снизу решетка $L$ вложима в решетку $\operatorname{Co}_{X}\left(\mathbb{D}_{\mathbb{D}}^{n}\right)$ для подходящих натурального $n>0$ и конечного $X \subseteq \mathbb{D}_{\mathbb{D}}^{n}$.

2.2. Выпуклые геометрии в упорядоченных множествах. Пусть $\langle P, \leqslant\rangle-$ частично упорядоченное множество. Подмножество $X \subseteq P$ называется выпуклым, если $x \leqslant z \leqslant y$ влечет $z \in X$ для любых $x, y \in X$ и любого $z \in P$. Для $X \subseteq P$ пусть $\operatorname{Co}(X)$ обозначает выпуклую оболочку множества $X$. Соответствующую решетку замкнутых (выпуклых) подмножеств мы обозначаем, как и для векторных пространств, через $\operatorname{Co}(P)$.

И вновь нетрудно видеть, что для любого частично упорядоченного множества $\langle P, \leqslant\rangle$ пространство замыкания $(P, \mathrm{Co})$ является выпуклой геометрией. Для произвольного класса $\mathscr{K}$ 
упорядоченных множеств пусть $\mathbf{C o}(\mathscr{K})$ обозначает соответствующий класс решеток выпуклых подмножеств. Пусть также $\mathscr{P}$ обозначает класс всех частично упорядоченных множеств.

В связи с постановкой проблемы 1 авторы работы [9] сочли интересным также выяснить, не будет ли класс конечных выпуклых геометрий вида $(P, \mathrm{Co})$ искомым универсальным классом для $\mathrm{SD}_{\mathrm{fin}}^{\vee}$. Поскольку квазимногообразие $\mathrm{SD}^{\vee}$ порождается своими конечными решетками (см. [9, теорема 4.1.7]), отрицательный ответ на поставленный вопрос дает следующая

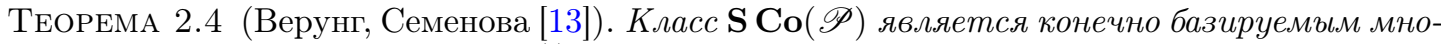
гообразием, поэтому $\mathbf{S} \mathbf{C o}(\mathscr{P}) \subset \mathrm{SD}^{\vee}$.

Отметим, что согласно характеризационной теореме 1.1 Мальцева класс $\mathbf{S} \mathbf{C o}(\mathscr{P})$ является квазимногообразием. Отметим также, что в работе [13] был найден конкретный конечный базис тождеств для многообразия $\mathbf{S ~} \mathbf{C o}(\mathscr{P})$.

В связи с теоремой 2.4 естественной представляется проблема описания решеток, принадлежащих классу $\mathbf{C o}(\mathscr{K})$ для различных конкретных классов упорядоченных множеств $\mathscr{K}$. Для произвольного натурального числа $n$ и для произвольного класса упорядоченных множеств $\mathscr{K}$ пусть $\mathscr{K}_{n}$ обозначает класс упорядоченных множеств из $\mathscr{K}$ высоты, не превосходящей $n$. Используя характеризационную теорему Мальцева, вновь нетрудно проверить, что класс $\mathbf{S ~ C o}\left(\mathscr{P}_{n}\right)$ является квазимногообразием для любого натурального $n$. Теорема 2.5 дает более детальную информацию об этом классе.

Теорема 2.5 (Верунг, Семенова [14]). (1) Kласс $\mathbf{S ~ C o}\left(\mathscr{P}_{n}\right)$ является конечно базируемым многообразием для любого натурального $n$.

(2) Многообразие $\mathbf{S ~ C o}\left(\mathscr{P}_{n}\right)$ локально конечно тогда и толъко тогда, когда $n<3$.

Отметим, что в работе [14] был найден конкретный конечный базис тождеств для многообразия $\mathbf{S} \mathbf{C o}\left(\mathscr{P}_{n}\right)$ для любого натурального $n$.

Пусть $\mathscr{L} \mathscr{O}$ обозначает класс линейно упорядоченных множеств (цепей). Вновь согласно характеризационной теореме Мальцева класс $\mathbf{S P} \mathbf{C o}(\mathscr{L} \mathscr{O})$ является квазимногообразием. Теорема 2.6 позволяет полностью описать решетку подквазимногообразий в $\mathbf{S P} \mathbf{C o}(\mathscr{L} \mathscr{O})$.

Теорема 2.6 (Верунг, Семенова [15]). (1) Класс $\mathbf{S P} \mathbf{C o}(\mathscr{L O})$ является конечно базируемым локально конечным многообразием.

(2) Любое подквазимногообразие в $\mathbf{S P} \mathbf{C o}(\mathscr{L O})$ является многообразием.

Решетка под(квази)многообразий в $\mathbf{S P} \mathbf{C o}(\mathscr{L} \mathscr{O})$. изображена на рис. 2 ; здесь $\operatorname{Co}(\mathbf{n})$ для всякого натурального $n>0$ обозначает решетку выпуклых подмножеств $n$-элементной цепи, а $\mathscr{B}_{n}$ обозначает интервал, изоморфный решетке подмножеств $n$-элементного множества. Конкретный конечный базис тождеств для многообразия $\mathbf{S P} \mathbf{C o}(\mathscr{L O})$ был найден в работе [15].

Лесом мы называем частично упорядоченное множество, в котором нижний конус, порожденный любым элементом, является цепью. Это условие равносильно также следующему: любые два несравнимых элемента не имеют верхней границы. Деревом мы называем лес, который является связным. Пусть $\mathscr{F}$ обозначает класс частично упорядоченных множеств, являющихся лесами, а $\mathscr{T}$ обозначает класс деревьев. И вновь характеризационная теорема Мальцева показывает, что класс $\mathbf{S ~ C o}(\mathscr{F})$ является квазимногообразием.

Теорема 2.7 (Замойска-Дженио, Семенова [16]). (1) Kлассъ $\mathbf{S ~ C o}(\mathscr{F})$ u $\mathbf{S ~ C o}(\mathscr{T})$ coвnaдают и образуют конечно базируемое многообразие.

(2) Класс $\mathbf{S ~} \mathbf{C o}\left(\mathscr{F}_{n}\right)$ является конечно базируемым многообразием для любого натурального $n$.

В связи с тем, что многообразие $\mathbf{S} \mathbf{C o}(\mathscr{L} \mathscr{O})$ локально конечно согласно теореме $2.6,(1)$ и, очевидно, имеет место включение $\mathscr{L} \mathscr{O} \subset \mathscr{T}$, интерес представляет следующая проблема. 


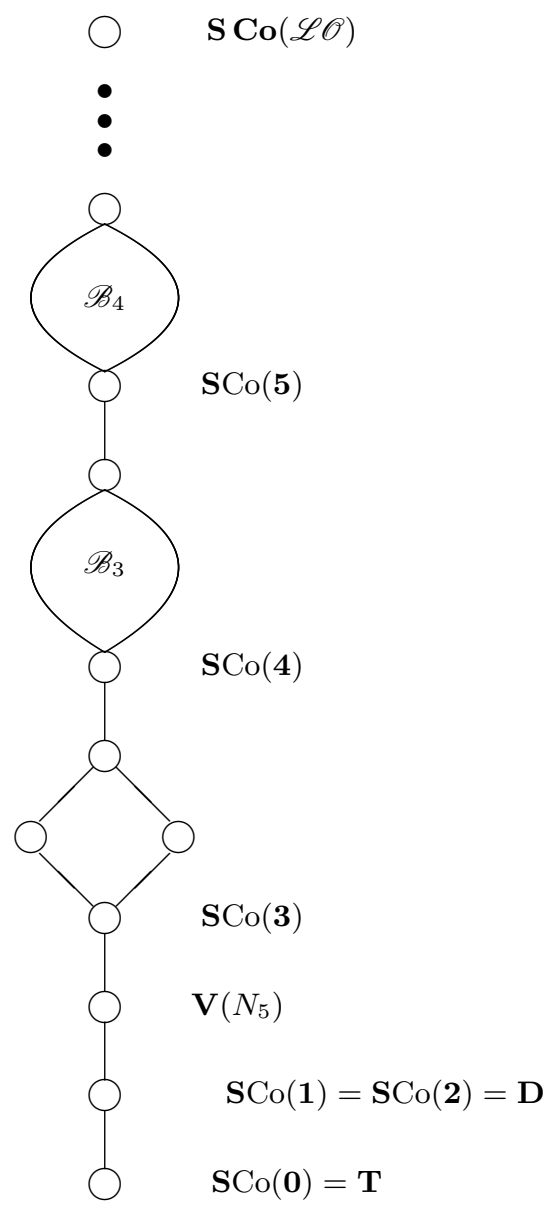

Рис. 2. Решетка под(квази)многообразий в $\mathbf{S ~ C o}(\mathscr{L O O}$

Проблема 3 (Замойска-Дженио, Семенова [16]). Является ли многообразие $\mathbf{S ~ C o}(\mathscr{T})$ локально конечным?

В теореме 2.8 и следствии 2.9 пусть $\mathscr{K}$ обозначает один из классов $\mathscr{P}, \mathscr{L} \mathscr{O}, \mathscr{T}$ или $\mathscr{P} n$, $\mathscr{F}_{n}$ для натурального $n$.

Теорема 2.8 (Верунг, Семенова [13]; Замойска-Дженио, Семенова [16]). Многообразие $\mathbf{S ~ C o}(\mathscr{K})$ порождается своими конечными решетками.

Таким образом, согласно теореме Маккинси (см. [17]) справедливо

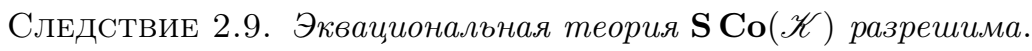

Согласно характеризационной теореме Мальцева класс $\mathbf{S P}(\operatorname{Co}(P))$ является квазимногообразием для любого конечного частично упорядоченного множества $\langle P, \leqslant\rangle$. Из результатов работы [15] вытекает, что если $\langle P, \leqslant\rangle$ - конечная цепь, то класс $\mathbf{S P}(\mathrm{Co}(P))$ является многообразием (см. рис. 2). Однако нам не известно, справедливо ли это утверждение в общем случае.

ПробЛема 4 (Верунг, Семенова [15]). Пусть $\langle P, \leqslant\rangle-$ конечное частично упорядоченное множество. Является ли класс $\mathbf{S P}(\mathrm{Co}(P))$ многообразием? 
Методы, развитые в работах [13]-[16], позволяют не только дать синтаксическую характеризацию классов решеток, вложимых в решетки выпуклых подмножеств упорядоченных множеств, принадлежащих различным классам, но также и охарактеризовать решетки, изоморфные таким решеткам. Ранее решетки, принадлежащие классу $\mathbf{C o}(\mathscr{P})$, были описаны в работе Биркгофа и Беннет [18]. Из описания этого класса, в частности, вытекает следующая

Теорема 2.10 (Биркгоф, Беннет [18]). Класс решеток $\mathbf{C o}(\mathscr{P})$ аксиоматизируем на языке первого порядка в классе всех непрерывных вверх решеток. Однако $\mathbf{C o}(\mathscr{P})$ не является конечно аксиоматизируемым в этом классе.

Тем не менее для решеток, изоморфных решеткам выпуклых подмножеств деревьев, справедлива

Теорема 2.11 (Замойска-Дженио, Семенова [19]). Kлассъ $\mathbf{C o}(\mathscr{F}), \mathbf{C o}\left(\mathscr{F}_{n}\right), n<\omega, u$ $\mathbf{C o}(\mathscr{L O})$ конечно аксиоматизируемы в классе всех непрерывных вверх решеток.

С упорядоченными множествами связан еще один класс выпуклых геометрий. Для частично упорядоченного множества $\langle P, \leqslant\rangle$ и для любого множества $X$ такого, что

$$
\{(a, a) \mid a \in P\} \subseteq X \subseteq \leqslant \subseteq P^{2},
$$

пусть $X^{\mathrm{tr}}$ обозначает транзитивное замыкание множества $X$; т.е. если $(a, b),(b, c) \in X$, то $(a, c) \in X$ для всех $a, b, c \in P$ и, таким образом, $\left\langle P, X^{\operatorname{tr}}\right\rangle$ является частично упорядоченным множеством. Нетрудно видеть, что пространство замыкания $\left(P^{2}, X^{\mathrm{tr}}\right)$ является выпуклой геометрией. Соответствующую решетку замкнутых подмножеств (которая называется также решеткой подпорядков) мы обозначаем через $\mathrm{O}(P, \leqslant)$. Кроме того, для произвольного класса $\mathscr{K}$ упорядоченных множеств пусть $\mathbf{O}(\mathscr{K})$ обозначает соответствующий класс решеток подпорядков.

Следующая теорема, доказанная Бредихиным и Шайном в [20] (другое доказательство в этого результата содержится в [21]), показывает, что класс решеток подпорядков универсален для класса всех решеток.

Теорема 2.12 (Бредихин, Шайн [20]). Любая решетка вложима в некоторую решетку подпорядков.

Что касается класса конечных решеток подпорядков, то согласно следующей теореме этот класс универсален для класса конечных ограниченных снизу решеток и поэтому не может служить кандидатом на роль класса, универсального для $\mathrm{SD}_{\mathrm{fin}}^{\vee}$ (см. проблему 1).

Теорема 2.13 (Сивак [22]). Решетка вложима в некоторую конечную решетку подпорядков тогда и только тогда, когда она конечна и органичена снизу.

Теорема 2.13 была передоказана в работе [21] и является следствием более общего результата, представленного в теореме 2.14 .

Теорема 2.14 (Семенова [21]). Класс $\mathbf{S O}\left(\mathscr{P}_{n}\right)$ является конечно базируемым многообразием для любого натурального $n$.

В работе [21] был найден конкретный конечный базис тождеств для многообразия $\mathbf{S O}\left(\mathscr{P}_{n}\right)$. Из доказательства теоремы 2.14 среди прочего вытекает такое

СледСтвиЕ 2.15. Решетка $\mathrm{O}(P, \leqslant)$ ограничена снизу тогда и толъко тогда, когда множество $\langle P, \leqslant\rangle$ имеет конечную высоту.

Согласно [12, теорема 2.84] многообразие всех решеток порождается конечными ограничнными снизу решетками. Поэтому в силу теоремы 2.13 справедливо такое

СлЕДСТВИЕ 2.16. Эквачиональная теория класса конечных решеток подпорядков совпадает с эквациональной теорией класса всех решеток. 
Из характеризационной теоремы Мальцева следует, что класс $\mathbf{S P}(\mathrm{O}(P, \leqslant))$ является квазимногообразием для любого конечного частично упорядоченного множества $\langle P, \leqslant\rangle$. Однако нам не известно решение следующей проблемы.

ПробЛема 5 (Семенова [21]). Пусть $\langle P, \leqslant\rangle$ - конечное частично упорядоченное множество. Является ли класс $\mathbf{S P}(\mathrm{O}(P, \leqslant))$ многообразием?

2.3. Выпуклые геометрии в теории квазимногообразий. Последний пример выпуклых геометрий, который будет нами рассмотрен, дают так называемые алгебраические подмножества полных решеток. Итак, пусть $L-$ полная решетка. Подмножество в $L$ называется алгебрачческим, если оно замкнуто в $L$ относительно произвольных пересечений и объединений по непустным направленным вверх подмножествам. Замкнутость относительно произвольных пересечений означает, в частности, что каждое алгебраическое подмножество в $L$ содержит наибольший элемент решетки $L$ и поэтому непусто. Для любого множества $X \subseteq L$ пусть $\operatorname{Sp}(X)$ обозначает наименьшее алгебраическое подмножество в $L$, содержащее $X$.

Полная решетка $L$ называется непрерьвной вверx, если в $L$ имеет место равенство

$$
a \wedge \bigvee D=\bigvee_{d \in D} a \wedge d
$$

для любого направленного вверх множества $D \subseteq L$ и любого $a \in L$. Двойственным образом определяются непрерывные вниз решетки. Если решетка $L$ непрерывна вверх, то решетка $\operatorname{Sp}(L)$ является непрерывной вниз и полудистрибутивной вверх (см., например, [9, теорема 4.1.1]). Кроме того, в этом случае для произвольных $A, B \in \operatorname{Sp}(L)$ выполняется равенство

$$
A \vee B=\{a \wedge b \mid a \in A, b \in B\} .
$$

Отсюда непосредственно следует, что пространство замыкания $(L, \mathrm{Sp})$ является выпуклой геометрией для любой непрерывной вверх решетки $L$. Соответствующую решетку замкнутых подмножеств (которая называется также решеткой алгебраических подмножеств) мы обозначаем через $\mathrm{Sp}(L)$. Кроме того, для произвольного подкласса $\mathscr{L}$ класса $\mathscr{U} \mathscr{C}$ всех полных непрерывных вверх решеток пусть $\mathbf{S p}(\mathscr{L})$ обозначает соответствующий класс решеток алгебраических подмножеств.

Хорошо известно, что любая алгебраическая решетка непрерывна вверх. Кроме того, согласно работе Горбунова и Туманова [23] (см. также [9, теорема 5.6.7]) для любой алгебраической решетки $L$ решека $\operatorname{Sp}(L)$ является коалгебраической и изоморфна решетке подквазимногообразий некоторого квазимногообразия чисто предикатной сигнатуры. Пусть $\mathscr{A}$ обозначает класс алгебраических решеток. В работе [23] была сформулирована следующая проблема.

Проблема 6 (Горбунов, Туманов [23]). Охарактеризовать решетки, принадлежащие классу $\mathbf{S} \mathbf{S p}(\mathscr{A})$. В частности, выяснить, верно ли, что $\mathbf{S} \mathbf{S p}(\mathscr{A})=\mathbf{S} \mathbf{S p}(\mathscr{U} \mathscr{C})$.

Хотя проблема 6 остается нерешенной, следует упомянуть один результат, к ней относящийся. Решетка $L$ называется непрерьвной по Cкотту (см. [24]), если $a=\bigvee\{x \in L \mid x \ll a\}$ для любого $a \in L$. Здесь запись $x \ll a$ означает, что для произвольного направленного вверх множества $D \subseteq L$, если $a \leqslant \bigvee D$, то $x \leqslant d$ для некоторого $d \in D$. Хорошо известно (см., например, [24]), что любая алгебраическая решетка непрерывна по Скотту, а любая непрерывная по Скотту решетка непрерывна вверх. Пусть $\mathscr{S} \mathscr{C}$ обозначает класс непрерывных по Скотту решеток.

Теорема 2.17 (Семенова [25]). Имеет место равенство $\mathbf{S} \mathbf{S p}(\mathscr{A})=\mathbf{S} \mathbf{S p}(\mathscr{S} \mathscr{C})$.

Близкие вопросы изучались в работе Верунга [26]. В частности, в этой работе было получено описание решеток, вложимых в непрерывные вниз полудистрибутивные вверх полные 
решетки. Отметим также, что для произвольной конечной решетки $L$ решетка $\operatorname{Sp}(L)$ изоморфна решетке нижних подполурешеток полурешетки, полученной из $L$ удалением наибольшего элемента. Несмотря на то, что решетки подполурешеток являются решетками замыканий выпуклых геометрий, нам будет более удобно рассмотреть их в контексте параграфа 4.

\section{3. Комбинаторные геометрии}

Пространство замыкания $(X, \varphi)$ называется комбинаторной геометрией, если оно обладает следующим свойством замены:

$$
a \in \varphi(Y \cup\{b\}) \quad \text { влечет } \quad b \in \varphi(Y \cup\{a\})
$$

для любых $a, b \in X$ и любого $Y \subseteq X$. Понятие комбинаторной геометрии служит обобщением понятия линейной зависимости векторов в векторных пространствах и является в некотором смысле антиподом понятия выпуклой геометрии. Таким образом, естественно ожидать, что и решетки замыканий комбинаторных геометрий будут отличаться по своим свойствам от решеток замыканий выпуклых геометрий. И это действительно так: решетки замыканий комбинаторных геометрий часто являются модулярными. Как было упомянуто выше, типичным примером решеток замыканий комбинаторных геометрий служат решетки подпространств векторных пространств.

Пусть $V_{\mathbb{D}}$ - векторное пространство над телом $\mathbb{D}$, и пусть $\operatorname{Sub}\left(V_{\mathbb{D}}\right)$ обозначает решетку подпространств в $V_{\mathbb{D}}$. Очевидно, $\operatorname{Sub}\left(V_{\mathbb{D}}\right)$ является решеткой замыканий комбинаторной геометрии $\left(V_{\mathbb{D}}, \mathrm{Sub}\right)$, где Sub обозначает оператор взятия линейной оболочки множества векторов. В общем случае решетка подпространств $\operatorname{Sub}\left(V_{\mathbb{D}}\right)$ является подпрямо неразложимой решеткой с относительными дополнениями и, кроме того, арговой, т.е. удовлетворяет аргову тождеству

$$
\forall x_{0}, x_{1}, x_{2}, y_{0}, y_{1}, y_{2} \quad \bigwedge_{i<3}\left(x_{i} \vee y_{i}\right) \leqslant\left(x_{0} \wedge\left(x_{1} \vee c\right)\right) \vee\left(y_{0} \wedge\left(y_{1} \vee c\right)\right)
$$

где

$$
c_{i}=\left(x_{j} \vee x_{k}\right) \wedge\left(y_{j} \vee y_{k}\right), \quad\{i, j, k\}=\{0,1,2\}, \quad c=\left(c_{0} \vee c_{1}\right) \wedge c_{2} .
$$

Отметим, что любая аргова решетка модулярна. Пусть $\mathbf{S u b}(\mathscr{V})$ обозначает класс решеток, изоморфных решеткам подпространств векторных пространств.

Если размерность пространства $V_{\mathbb{D}}$ конечна, то решетка $\operatorname{Sub}\left(V_{\mathbb{D}}\right)$ простая и имеет конечную высоту (см., например, книгу Биркгофа [6]). Согласно следующей координатизационной теореме фон Ноймана-Йонссона справедливо и частичное обращение последнего утверждения.

Теорема 3.1 (фон Нойман [27]; Йонссон [28]). Пусть L - простая аргова решетка с дополнениями конечной высоты $n \geqslant 3$. Тогда $L \cong \operatorname{Sub}\left(\mathbb{D}_{\mathbb{D}}^{n}\right)$ для некоторого тела $\mathbb{D}$.

Известной нерешенной проблемой является следующая

ПроБлема 7 (Дилуорс [6]). Является ли класс решеток, вложимых в модулярные решетки с относительными дополнениями, многообразием?

Согласно характеризационной теореме Мальцева класс решеток, о котором идет речь в проблеме 7 , является квазимногообразием. Следующий результат Йонссона связывает проблему 7 с решетками подпространств, характеризуя решетки, вложимые в арговы решетки с (относительными) дополнениями.

ТЕОРема 3.2 (Йонссон [28]). Для модулярной решетки L с относительными дополнениями равносильны следующие условия:

(1) $L \in \mathbf{S}(\mathrm{Cl}(X, \varphi))$ для некоторой проективной геометрии $(X, \varphi)$;

(2) $L \in \mathbf{S}(\operatorname{Sub}(A))$ для некоторой абелевой группь $A$;

(3) $L \in \mathbf{S P}(\mathbf{S u b}(\mathscr{V}))$.

(4) L аргова. 
3.1. Э-многообразия. Всюду далее до конца параграфа 3 нам будет удобно рассматривать решетки в сигнатуре $\{\vee, \wedge, 0\}$, где 0 будет интерпретироваться как наименьший элемент решетки. Кроме того, если $\Lambda$ - коммутативное кольцо с единицей, то $\Lambda$-алгебры будут рассматриваться нами в сигнатуре $\{\Lambda,+,-, \cdot, 0\}$. При этом кольца будут рассматриваться нами как $\mathbb{Z}$-алгебры. Рассмотрим следующие предложения:

$$
\begin{aligned}
\varphi_{l}: & \forall x y \quad \exists z \quad[x \wedge y \wedge z=0] \&[(x \wedge y) \vee z=x] \\
\varphi_{a}: & \forall x \quad \exists y \quad[x y x=x] .
\end{aligned}
$$

Решетка $L$ называется решеткой с относительными дополнениями, если $L=\varphi_{l}$. Пусть $\mathscr{M}$ обозначает класс модулярных решеток с относительными дополнениями. Хорошо известно, что модулярная решетка с наибольшим элементом имеет дополнения тогда и только тогда, когда она имеет относительные дополнения (см., например, [6]).

$\Lambda$-алгебра $R$ называется регулярной, если $R=\varphi_{a}$. Далее $\mathscr{R}_{\Lambda}$ обозначает класс регулярных $\Lambda$-алгебр, а $\mathscr{R}$ - класс регулярных колец. Хорошо известно, что в регулярных кольцах любой конечно порожденный односторонний идеал порождается идемпотентом (см., например, Скорняков [29]). Таким образом, если рассматривать регулярные кольца как $\mathbb{Z}$-алгебры, то конечно порожденные односторонние идеалы колец - это в точности подалгебры. Пусть $\mathbb{L}(R)$ обозначает решетку конечно порожденных (правых) идеалов регулярного кольца $R$. Решетка $\mathbb{L}(R)$ является модулярной решеткой с относительными дополнениями. Если же кольцо $R$ артиново, то $\mathbb{L}(R)$ является модулярной решеткой с дополнениями конечной высоты.

Для произвольного кольца $R$ и $n>0$ через $R^{n \times n}$ мы обозначаем кольцо матриц порядка $n \times n$ над $R$. Если кольцо $R$ коммутативно и содержит единицу, то кольцо $R^{n \times n}$ можно рассматривать как $R$-алгебру. Хорошо известно, что если кольцо $R$ регулярно, то и кольцо матриц $R^{n \times n}$ регулярно. Кроме того, кольцо эндоморфизмов $\operatorname{End}\left(V_{\mathbb{D}}\right)$ векторного пространства $V_{\mathbb{D}}$ над телом $\mathbb{D}$ всегда регулярно и $\operatorname{Sub}\left(V_{\mathbb{D}}\right) \cong \mathbb{L}\left(\operatorname{End}\left(V_{\mathbb{D}}\right)\right)$.

Пусть далее $\mathscr{K}=\mathscr{M}$ либо $\mathscr{K}=\mathscr{R}_{\Lambda}$ для некоторого коммутативного кольца $\Lambda$ с единицей. Определим оператор $\mathbf{S}_{\exists}$, полагая для $\mathscr{K}^{\prime} \subseteq \mathscr{K}$

$$
\mathbf{S}_{\exists}\left(\mathscr{K}^{\prime}\right)=\mathbf{S}\left(\mathscr{K}^{\prime}\right) \cap \mathscr{K} .
$$

Класс $\mathscr{K}^{\prime} \subseteq \mathscr{K}$ называется $\exists$-многообразием, если $\mathscr{K}^{\prime}=\mathbf{X}\left(\mathscr{K}^{\prime}\right)$ для любого $\mathbf{X} \in\left\{\mathbf{H}, \mathbf{S}_{\exists}, \mathbf{P}\right\}$.

Теорема 3.3 (Херрман, Семенова [30]). Для любого класса $\mathscr{K}^{\prime} \subseteq \mathscr{K}$ выполняются следующие утверждения:

(1) $\mathbf{V}_{\exists}(\mathscr{K})=\mathbf{H S}_{\exists} \mathbf{P}(\mathscr{K})$ есть наименъшее $\exists$-многообразие, содержащее $\mathscr{K}$;

(2) класс подпрямо неразложимых алгебр из $\mathbf{V}_{\exists}(\mathscr{K})$ содержится в классе $\mathbf{H S}_{\exists} \mathbf{P}_{u}(\mathscr{K})$;

(3) любое $\exists$-многообразие порождается своими конечно порожденными подпрямо неразложимыми алгебрами.

Из теоремы 3.3 вытекает, что в Э-многообразиях существуют свободные алгебры. Кроме того, в силу замкнутости относительно гомоморфных образов $\exists$-многообрзия аксиоматизируются позитивными предложениями. С другой стороны, они, очевидно, аксиоматизируются хорновыми предложениями. Не известно решение следующей проблемы.

Проблема 8 (Херрман, Семенова [30]). Может ли каждое $\exists$-многообразие быть аксиоматизировано позитивными хорновыми предложениями (иными словами, тождествами)?

3.2. Регулярные кольца. Согласно теореме $3.3,(3)$ всякое Э-многообразие $\Lambda$-алгебр порождается своими конечно порожденными подпрямо неразложимыми алгебрами. Теорема 3.4 и следствие 3.5 конкретизируют это утверждение.

Теорема 3.4 (Херрман, Семенова [30]). $\exists$-многообразие регулярных $\Lambda$-алгебр $\mathscr{R}_{\Lambda}$ порождается алгебрами вида $\mathbb{F}^{n \times n}$, где $n>1$ u $\mathbb{F}$ - бакторполе кольца $\Lambda$. 
СлеДСТВИЕ 3.5. Любое Э-многообразие регулярных $\Lambda$-алгебр порождается своими простыми артиновыми алгебрами.

СлЕДСТВиЕ 3.6. Свободные регулярные $\Lambda$-алгебры резидуалъно артиновы.

Отметим что утверждение следствия 3.6 было доказано для алгебр с единицей в работе Гудерла, Менала и Монкаси [31]. Для произвольного простого числа $p$ пусть $\mathbb{F}_{p}$ обозначает поле из $p$ элементов. Теорема 3.4 в случае регулярных колец выглядит так.

СледСтвиЕ 3.7 (Херрман, Семенова [30]). Имеем:

(1) $\mathscr{R}=\mathbf{V}_{\exists}\left(\mathbb{F}_{p}^{n \times n} \mid m<n<\omega\right.$, простое $)$ для произвольного натурального $m$;

(2) свободные регулярные кольца резидуалъно конечны;

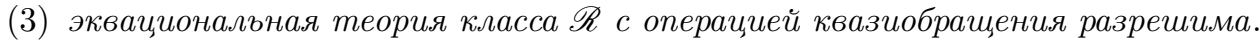

3.3. Модулярные решетки с дополнениями. Согласно теореме $3.3,(3)$ всякое $\exists$-многообразие модулярных решеток с относительными дополнениями порождается своими конечно порожденными подпрямо неразложимыми решетками. Теорема 3.4 и следствие 3.5 позволяют утверждать большее.

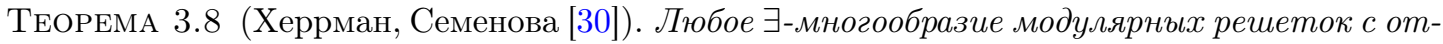
носительными дополнениями порождается своими простыми решетками конечной высоmbl.

СледствиЕ 3.9 (Херрман, Семенова [30]). Имеем:

(1) для произвольного натурального $m$ многообразие арговых решеток с относительными дополнениями порождается решетками $\mathbb{L}\left(\mathbb{F}_{p}^{n \times n}\right)$, где $n>m$ u $p$ простое;

(2) свободные арговы решетки с относительными дополнениями резидуально конечны;

(3) эквачиональная теория класса арговых (модулярных) решеток с операцией относительного дополнения разрешима.

Хорошо известно (см. [6], [7]), что решетка $L$ и ее решетка идеалов $\operatorname{Id}(L)$ порождают одно и то же многообразие, т.е. $\mathbf{V}(L)=\mathbf{V}(\operatorname{Id}(L))$. Возвращаясь к открытой проблеме 7 , отметим такое следствие теоремы 3.3.

СледСтвиЕ 3.10 (Херрман, Семенова [30]). Если решетка L вложима в некоторую модулярную решетку с относительными дополнениями, то то же самое верно и для ее решетки идеалов $\operatorname{Id}(L)$.

\section{4. Другие пространства замыкания}

Напомним, что для произвольной алгебры $A$ решетка подалгебр $\operatorname{Sub}(A)$ алгебры $A$ есть решетка замыканий пространства замыкания $(A, \mathrm{Sub})$. Для произвольного класса алгебр $\mathscr{K}$ пусть $\operatorname{Sub}(\mathscr{K})$ обозначает класс решеток подалгебр алгебр из $\mathscr{K}$.

4.1. Решетки под(полу)групп. Классический результат Уитмена 1946 г. утверждает, что класс решеток подгрупп универсален для класса всех решеток.

Теорема 4.1 (Уитмен [32]). Любая решетка вложима в решетку подгрупп некоторой гpynnы.

Что касается конечной версии теоремы 4.1, то вопрос о том, вложима ли любая конечная решетка в решетку подгрупп некоторой конечной группы, долго оставался открытым. Он был положительно решен в известной работе Пудлака и Тумы 1980 г. [33].

Теорема 4.2 (Пудлак, Тума [33]). Любая конечная решетка вложима в решетку подгрупп некоторой конечной группы.

В работе [34] Тума получил следующее уточнение теоремы Уитмена. 
Теорема 4.3 (Тума [34]). Решетка является алгебраической тогда и только тогда, когда она изоморфна интервалу в решетке подгрупп некоторой группы.

Другое доказательство теоремы 4.3 можно найти в работе Репницкого [35]. Заметим, что утверждение о том, что любая конечная решетка изоморфна интервалу в решетке подгрупп некоторой конечной группы, согласно работе Палфи и Пудлака [36] эквивалентно тому, что любая конечная решетка изоморфна решетке конгруэнций некоторой конечной алгебры конечной сигнатуры. Вопрос об истинности последнего утверждения является известной открытой проблемой. Отметим также, что недавно Репницкий и Тума доказали в [37], что любая алгебраическая решетка с не более чем счетным числом компактных элементов вложима как интервал в решетку подгрупп некоторой локально конечной группы.

Из теоремы 4.1, в частности, следует, что и класс решеток подполугрупп универсален, т.е. любая решетка вложима также в решетку подполугрупп некоторой полугруппы. Репницкий доказал более сильные утверждения. Пусть $\mathscr{S} \mathscr{L}$ обозначает класс всех полурешеток (т.е. класс всех коммутативных идемпотентных полугрупп), пусть $\mathscr{S}^{\prime}$ обозначает класс всех коммутативных полугрупп без идемпотентов с сокращением и однозначным извлечением корня, пусть $\mathscr{C} \mathscr{N}_{2}$ обозначает класс всех коммутативных 2-нильполугрупп, т.е. полугрупп с нулем, удовлетворяющих тождеству

$$
\forall x \quad x^{2}=0 .
$$

Теорема 4.4 (Репницкий [38]). Пусть $\mathscr{S} \in\left\{\mathscr{S} \mathscr{L}, \mathscr{S}^{\prime}, \mathscr{C} \mathscr{N}_{2}\right\}$. Для любой решетки L cуществует полугруппа $S \in \mathscr{S}$ такая, что $L$ вложима в $\operatorname{Sub}(S)$.

При доказательстве этой теоремы в [38] Репницкий существенным образом использовал теорему Бредихина-Шайна об универсальности решеток подпорядков (теорема 2.12). Более точно, он показал, что любая решетка подпорядков вложима в решетку подполугрупп для подходящей полугруппы, принадлежащей соответствующему классу. В связи с этим Шевриным и Овсянниковым был поставлен вопрос (см. [39, вопрос VIII.7]) о существовании доказательства теоремы 4.4, которое не использовало бы решетки подпорядков. Такое доказательство было найдено в работах автора [40]-[42]. Это доказательство использует технику раскрашенных деревьев, развитую в работах Верунга и автора [10], [13]-[15], а также в работе автора [21].

4.2. Свободные полугруппы. Универсальность класса конечных решеток подполугрупп для класса конечных ограниченных снизу решеток вытекает из следующего результата Репницкого, который дополняет теорему 2.13.

Теорема 4.5 (Репницкий [43]). Для конечной решетки L равносилъны следующие условия:

(1) L вложима в решетку подполугрупп (конечно порожденной) свободной (коммутативной) полугруппъ;

(2) L вложима в решетку подполугрупп (конечно порожденной) свободной (коммутативной) 2-нильполугруппы;

(3) L вложима в решетку подполугрупп (конечно порожденной) свободной полурешетки;

(4) L вложима в решетку подполугрупп конечной нильпотентной полугруппь;

(5) L ограничена снизу.

Пусть $\mathscr{S}_{F}$ обозначает класс всех свободных полугрупп; $\mathscr{S}_{F C}-$ класс всех свободных коммутативных полугрупп; $\mathscr{S}_{F N 2}-$ класс всех свободных 2-нильполугрупп; $\mathscr{S}_{F C N 2}-$ класс всех свободных коммутативных 2-нильполугрупп; $\mathscr{S}_{F S L}-$ класс всех свободных полурешеток. На языке операторов теорема 4.5 выглядит следующим образом. 
СлЕДСТВИЕ 4.6 (Репницкий [43]). Справедлива иепочка равенств

$$
\begin{aligned}
\operatorname{SSub}\left(\mathscr{S}_{F}\right)_{\mathrm{fin}} & =\operatorname{SSub}\left(\mathscr{S}_{F C}\right)_{\mathrm{fin}}=\operatorname{SSub}\left(\mathscr{S}_{F N 2}\right)_{\mathrm{fin}}=\operatorname{SSub}\left(\mathscr{S}_{F C N 2}\right)_{\mathrm{fin}} \\
& =\operatorname{SSub}\left(\mathscr{S}_{F S L}\right)_{\mathrm{fin}}=L B_{\mathrm{fin}} .
\end{aligned}
$$

Из этого результата, в частности, вытекает аналог следствия 2.16 для различных классов конечных полугрупп.

СлЕДСТВИЕ 4.7. Эквациональная теория класса решеток подполугрупп конечно порожденных свободных коммутативных 2-нильполугрупп, конечно порожденных свободных полурешеток, а также конечных нильпотентных полугрупп совпадает с эквачиональной теорией класса всех решеток.

В связи с результатом, установленным в следствии 4.6, в монографии Шеврина и Овсянникова была поставлена проблема (см. [39, проблема 28.14.1]) о совпадении классов произвольных решеток, вложимых в решетки подполугрупп свободных полугрупп, принадлежащих одному из упомянутых выше классов. Положительное решение этой проблемы вытекает из следующей теоремы, которая обобщает теорему 4.5 .

Теорема 4.8 (Семенова [44]). Для решетки L и для бесконечного кардинала к равносильнъи следующие условия:

(1) L вложима в решетку подполугрупп свободной полугруппь ранга к;

(2) L вложима в решетку подполугрупп свободной коммутативной полугруппы ранга к;

(3) L вложима в решетку подполугрупп свободной 2-нильполугруппы ранга к;

(4) L вложима в решетку подполугрупп свободной коммутативной 2-нильполугруппь ранга $\kappa$;

(5) L вложима в решетку подполугрупп свободной полурешетки ранга к;

(5) $L \in \mathbf{S P}_{\kappa}\left(L B_{\text {fin }}\right)$.

На языке операторов теорема 4.8 выглядит следующим образом.

СлЕДСтвиЕ 4.9 (Семенова [44]). Справедлива иепочка равенств

$$
\operatorname{SSub}\left(\mathscr{S}_{F}\right)=\operatorname{SSub}\left(\mathscr{S}_{F C}\right)=\operatorname{SSub}\left(\mathscr{S}_{F N 2}\right)=\operatorname{SSub}\left(\mathscr{S}_{F C N 2}\right)=\operatorname{SSub}\left(\mathscr{S}_{F S L}\right)=\operatorname{SP}\left(L B_{\text {fin }}\right),
$$

и этот класс не аксиоматизируем на языке первого порядка.

Теорема 4.8 описывает решетки, вложимые в решетки подполугрупп свободных полугрупп бесконечного ранга. Что можно сказать о свободных полугруппах конечного ранга? Поскольку любая конечно порожденная полурешетка и любая конечно порожденная коммутативная 2-нильполугруппа конечны, класс решеток, вложимых в них подполугрупп, совпадает с классом всех конечных ограниченных снизу решеток в силу следствия 4.6. Для свободных полугрупп справедлив такой результат.

СледСтвиЕ 4.10 (Семенова [44]). Для решетки L и для натурального $n>1$ равносильны следующие условия:

(1) L вложима в решетку подполугрупп свободной полугруппь ранга $n$;

(2) L вложима в решетку подполугрупп свободной полугруппы счетного ранга;

(3) $L \in \mathbf{S P}_{\omega}\left(L B_{\text {fin }}\right)$.

Однако ничего не известно для свободных коммутативных полугрупп и свободных 2-нильполугрупп конечного ранга. Для натурального $n>1$ пусть $F C(n)$ обозначает свободную коммутативную полугруппу ранга $n$, а $F N(n)$ - свободную 2-нильполугруппу ранга $n$.

Проблема 9 (Семенова [44]). Пусть $m, n>1$ - различные натуральные числа.

(1) Описать классы решеток, вложимых в решетку $\operatorname{Sub}(F C(n))$ и в решетку $\operatorname{Sub}(F N(n))$. 
Совпадают ли эти классы? Являются ли они аксиоматизируемыми? Являются ли они (квази)многообразиями?

(2) Совпадают ли классы решеток, вложимых в решетки $\operatorname{Sub}(F C(m))$ и $\operatorname{Sub}(F C(n))$ ?

(3) Совпадают ли классы решеток, вложимых в решетки $\operatorname{Sub}(F N(m))$ и $\operatorname{Sub}(F N(n))$ ?

4.3. Нильпотентные полугруппы. Пусть $m>0$. Полугруппа с нулем называется m-нильпотентной, если она удовлетворяет тождеству

$$
\forall x_{0}, \ldots, x_{m-1} \quad x_{0} \cdots x_{m-1}=0 .
$$

В проблеме 28.14.2 из монографии Шеврина и Овсянникова [39] ставился вопрос об описании класса решеток, вложимых в решетки подполугрупп (конечных) $m$-нильпотентных полугрупп. Следующий результат дает синтаксическое описание этого класса решеток, одновременно уточняя теорему 2.14 .

Теорема 4.11 (Семенова [42]). Для решетки L и для натуралъного $m$ равносилъны следующие условия:

(1) L вложима в решетку подполугрупп некоторой (коммутативной $)(m+1)$-нильпотентной полугруппы;

(2) L вложима в решетку подполугрупп некоторой (коммутативной $)(m+1)$-нильпотентной 2-нильполугруппы;

(3) L вложима в решетку подпорядков некоторого частично упорядоченного множества высоты, не превосходящей $m$.

В частности, справедливы такие утверждения.

СледСтвиЕ 4.12 (Семенова [42]). Для всякого положительного натурального $m$ класс решеток, вложимых в решетки подполугрупп m-нильпотентных полугрупп, содержится в классе ограниченных снизу решеток и образует конечно базируемое многообразие.

СлЕДСТвиЕ 4.13 (Семенова [42]). Для конечной решетки L и для натуралъного т равносильны следующие условия:

(1) L вложима в решетку подполугрупп некоторой $($ коммутативной $)(m+1)$-нильпотентной полугруппь;

(2) L вложима в решетку подполугрупп некоторой конечной $($ коммутативной) $(m+1)$ нильпотентной полугруппы;

(3) L вложима в решетку подпорядков некоторого частично упорядоченного множества высоты, не превосходящей $m$;

(4) L вложима в решетку подпорядков некоторого конечного частично упорядоченного множества высоты, не превосходящей $m$.

Таким образом, класс решеток, вложимых в решетки подполугрупп конечных $m$-нильпотентных полугрупп, аксиоматизируется конечным числом тождеств в классе всех конечных решеток и поэтому образует псевдомногообразие.

4.4. Полурешетки. В связи с тем, что класс решеток, вложимых в решетки подполурешеток конечных полурешеток, совпадает с классом конечных ограниченных снизу решеток согласно следствию 4.6, естественным является вопрос о том, нельзя ли сузить класс конечных полурешеток, налагая на полурешетки те или иные требования так, чтобы класс решеток их подполурешуток оставался универсальным для класса конечных ограниченных снизу решеток. Следующий результат показывает, что требование на полурешетку быть деревом, является слишком сильным для этой цели.

Теорема 4.14 (Семенова [45]). Для конечной решетки L равносильны следующие условия:

(1) L вложима в решетку подполурешеток некоторой полурешетки, чья диаграмма Хассе является деревом; 
(2) L вложима в решетку подполурешеток некоторой конечной полурешетки, чъя диаграмма Хассе является деревом.

Более того, класс решеток, вложимых в решетки подполурешеток конечных деревъев, аксиоматизируется тождествами в классе всех конечных решеток и поэтому образует псевдомногообразие.

Автор благодарит программный комитет Международной конференции "Мальцевские чтения 2009" за предоставленную возможность выступить с докладом на конференции.

\section{Список литературы}

[1] А. Мальцев, “О включении ассоциативных систем в группы”, Матем. сб., 6:2 (1939), 331-336.

[2] А. Мальцев, “О включении ассоциативных систем в группы. II", Матем. сб., 8(50):2 (1940), $251-264$.

[3] А. И. Мальцев, "Несколько замечаний о квазимногообразиях алгебраических систем", Алгебра и логика, 5:3 (1966), 3-9.

[4] А.И. Мальцев, Алгебраические системы, Современная алгебра, Наука, М., 1970; А. I. Mal'cev, Algebraic Systems, Grundlehren Math. Wiss., 192, Springer-Verlag, Berlin, 1973.

[5] G. Birkhoff, "On the structure of abstract algebras", Proc. Cambridge Philos. Soc., 31 (1935), $433-454$.

[6] G. Birkhoff, Lattice Theory, Amer. Math. Soc. Colloq. Publ., 25, Amer. Math. Soc., Providence, RI, 1967; Г. Биркгоф, Теория решеток, Наука, М., 1984.

[7] G. Grätzer, General Lattice Theory, Birkhäuser Verlag, Basel, 1998.

[8] B. Korte, L. Lovász, R. Schrader, Greedoids, Algorithms Combin., 4, Springer-Verlag, Berlin, 1991.

[9] В. А. Горбунов, Алгебраическая теория квазимногообразий, Сибирская школа алгебры и логики, Научная книга, Новосибирск, 1999; V.А. Gorbunov, Algebraic Theory of Quasivarieties, Siberian School Algebra Logic, Consultants Bureau, New York, NY, 1998.

[10] Ф. Верунг, М.В. Семёнова, "Подрешетки решеток выпуклых подмножеств векторных пространств", Алгебра и логика, 43:3 (2004), 261-290.

[11] R. N. McKenzie, "Equational bases and nonmodular lattice varieties", Trans. Amer. Math. Soc., 174 (1972), 1-43.

[12] R. Freese, J. Ježek, J. B. Nation, Free Lattices, Math. Surveys Monogr., 42, Amer. Math. Soc., Providence, RI, 1995.

[13] M. Semenova, F. Wehrung, "Sublattices of lattices of order-convex sets, I. The main representation theorem", J. Algebra, 277:2 (2004), 825-860.

[14] M. Semenova, F. Wehrung, "Sublattices of lattices of order-convex sets, II. Posets of finite length", Internat. J. Algebra Comput., 13:5 (2003), 543-564.

[15] M. Semenova, F. Wehrung, "Sublattices of lattices of order-convex sets, III. The case of totally ordered sets", Internat. J. Algebra Comput., 14:3 (2004), 357-387.

[16] M. V. Semenova, A. Zamojska-Dzienio, "On lattices embeddable into lattices of order-convex sets. Case of trees", Internat. J. Algebra Comput., 17:8 (2007), 1667-1712.

[17] J.C. C. McKinsey, "The decision problem for some classes of sentences without quantifiers", J. Symbolic Logic, 8:3 (1943), 61-76.

[18] G. Birkhoff, M. K. Bennett, "The convexity lattice of a poset", Order, 2:3 (1985), 223-242.

[19] M. Semenova, A. Zamojska-Dzienio, "Lattices of order-convex sets of forests", Order, 27:3 (2010), 383-404.

[20] D. Bredihin, B. Schein, "Representation of ordered semigroups and lattices by binary relations", Colloq. Math., 39:1 (1978), 1-12.

[21] М. В. Семенова, "О решётках, вложимых в решётки подпорядков", Алгебра и логика, 44:4 (2005), 483-511.

[22] B. Sivák, "Representation of finite lattices by orders on finite sets", Math. Slovaca, 28:2 (1978), 203-215. 
[23] В.А. Горбунов, В. И. Туманов, "Об одном классе решеток квазимногообразиий”, Алгебра и логика, 19:1 (1980), 59-80.

[24] G. Gierz, K. H. Hofmann, K. Keimel, J. D. Lawson, M. Mislove, D. S. Scott, Continuous Lattices and Domains, Encyclopedia Math. Appl., 93, Cambridge Univ. Press, Cambridge, 2003.

[25] M. Semenova, "On lattices embeddable into lattices of algebraic subsets", Algebra Universalis, 61:3-4 (2009), 399-405.

[26] F. Wehrung, "Sublattices of complete lattices with continuity conditions", Algebra Universalis, 53:2-3 (2005), 149-173.

[27] J. von Neumann, Continuous Geometries, Princeton Math. Ser., 25, Princeton Univ. Press, Princeton, NJ, 1960.

[28] B. Jónsson, "Representations of complemented modular lattices", Trans. Amer. Math. Soc., 97 (1960), 64-94.

[29] Л. А. Скорняков, Дедекиндовы структуръ с дополнениями и регулярнъе кольца, Современные проблемы математики, Физматгиз, M., 1961; L. A. Skornyakov, Complemented Modular Lattices and Regular Rings, Oliver \& Boyd, Edinburgh-London, 1964.

[30] C. Herrmann, M. Semenova, "Existence varieties of regular rings and complemented modular lattices", J. Algebra, 314:1 (2007), 235-251.

[31] K. R. Goodearl, P. Menal, J. Moncasi, "Free and residually Artinian regular rings", J. Algebra, 156:2 (1993), 407-432.

[32] Ph. M. Whitman, "Lattices, equivalence relations, and subgroups", Bull. Amer. Math. Soc., 52 (1946), $507-522$.

[33] P. Pudlák, J. Tůma, "Every finite lattice can be embedded in a finite partition lattice", Algebra Universalis, 10:1 (1980), 74-95.

[34] J. Tůma, "Intervals in subgroup lattices of infinite groups", J. Algebra, 125:2 (1989), 367-399.

[35] V.B. Repnitskiř, "A new proof of Tůma's theorem on intervals in subgroup lattices", Contributions to General Algebra, 16, Heyn, Klagenfurt, 2005, 213-230.

[36] P. P. Pálfy, P. Pudlák, "Congruence lattices of finite algebras and intervals in subgroup lattices of finite groups", Algebra Universalis, 11:1 (1980), 22-27.

[37] V. B. Repnitski1̌, J. Tůma, On Intervals in Subgroup Lattices of Locally Finite Groups, Manuscript, 2006.

[38] В. Б. Репницкий, "О представлении решеток решетками подполугрупп”, Изв. вузов. Матем., 1996, № 1, 60-70.

[39] L. N. Shevrin, A. Ja. Ovsyannikov, Semigroups and their Subsemigroup Lattices, Math. Appl., 379, Kluwer Acad. Publ., Dordrecht, 1996.

[40] М. В. Семёнова, "О решётках, вложимых в решётки подполугрупп. І. Полурешётки", Алгебра u логика, 45:2 (2006), 215-230.

[41] М. В. Семёнова, "О решётках, вложимых в решётки подполугрупп. II. Полугруппы с сокращением", Алгебра и логика, 45:4 (2006), 436-446.

[42] М.В. Семёнова, "О решетках, вложимых в решетки подполугрупп. III. Нильпотентные полугруппы”, Сиб. матем. журн., 48:1 (2007), 192-204.

[43] V. B. Repnitskiǐ, "On finite lattices embeddable in subsemigroup lattices", Semigroup Forum, 46:1 (1993), 388-397.

[44] M. V. Semenova, "On lattices embeddable into subsemigroup lattices. IV. Free semigroups", Semigroup Forum, 74:2 (2007), 191-205.

[45] М. В. Семёнова, "О решетках, вложимых в решетки подполугрупп. V. Деревья", Сиб. матем. журн., 48:4 (2007), 894-913. 